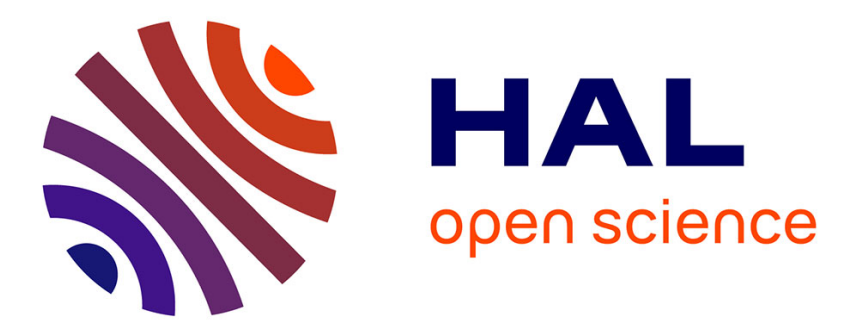

\title{
Acculturation, mutation des pratiques sexuelles et nouveaux regards sur la beauté en Afrique: le cas du Sénégal, du Cameroun et du Burkina Faso
}

Emmanuel Cohen, Amadou Ndao, Gilles Boëtsch

\section{- To cite this version:}

Emmanuel Cohen, Amadou Ndao, Gilles Boëtsch. Acculturation, mutation des pratiques sexuelles et nouveaux regards sur la beauté en Afrique: le cas du Sénégal, du Cameroun et du Burkina Faso. CORPS : Revue Interdisciplinaire, 2018, N¹6, pp.243-256. 10.3917/corp1.016.0243 . hal-03455930

\author{
HAL Id: hal-03455930 \\ https://hal.science/hal-03455930
}

Submitted on 8 Dec 2021

HAL is a multi-disciplinary open access archive for the deposit and dissemination of scientific research documents, whether they are published or not. The documents may come from teaching and research institutions in France or abroad, or from public or private research centers.
L'archive ouverte pluridisciplinaire HAL, est destinée au dépôt et à la diffusion de documents scientifiques de niveau recherche, publiés ou non, émanant des établissements d'enseignement et de recherche français ou étrangers, des laboratoires publics ou privés. 


\section{ACCULTURATION, MUTATION DES PRATIQUES SEXUELLES ET NOUVEAUX REGARDS SUR LA BEAUTE EN AFRIQUE : le cas du Sénégal, du Cameroun et du Burkina Faso}

Emmanuel Cohen Amadou Ndao Gilles Boëtsch

\section{ETAT DES LIEUX SUR LE CORPS ET LA SEXUALITE EN AFRIQUE}

Les études sur la sexualité en Afrique subsaharienne montrent vis-à-vis de la sexualité un intérêt «traditionnel » important porté à la dimension reproductive. Contrairement à ce qui se passe dans les sociétés industrielles, la sexualité dans de nombreuses traditions de populations africaines n'est pas constituée en un domaine autonome, indépendant de la reproduction (Warnier, 1993 : 124). La taille de la famille, le nombre d'enfants, seraient les premiers critères observés dans les traditions de ces populations pour juger de la bonne santé d'un couple marié. En effet, le rôle des enfants est indispensable dans le fonctionnement d'une société où le développement technique agricole est faible (Coquery-Vidrovitch, 1988 : 67). De par ce constat, Ndongo A Pitshandenge (1994 : 55) explique qu'« en Afrique, les enfants étaient et demeurent considérés comme le meilleur des biens ». Ainsi, les sociétés africaines ont toujours considérés la procréation comme l'objectif fondamental du mariage, ceci entraînant des codes de conduites, transmis par l'éducation, faisant la promotion de la maternité et de la paternité. La féminité serait d'abord associée à la fertilité, «ce sont les capacités reproductrices de la femme qui déterminent le statut auquel elle peut prétendre. [...] Chez les Mossi, une femme sans enfant est une "femme vide ». [...] Il est même fréquent d'entendre dire qu'une femme " a soit un enfant au dos, soit un enfant dans le ventre» (Desjeux et al., 1983, n.p) ». A l'Ouest Cameroun, le régime polygame est basé sur le devoir/prestige de maximiser sa descendance pour les hommes les plus « aptes ». Les notables sont les «hommes puissants », virils par excellence, détenant suffisamment de force vitale pour pouvoir féconder (Warnier, 1993 : 131-132).

Pour autant, les études sur la modernisation de l'Afrique montrent qu'une affirmation du plaisir face à la reproduction dans la vie sexuelle s'insère progressivement dans les grandes villes africaines (Ngondo A Pitshandenge, 1994 : 59-60 ; Amouzou 2009 : 43). Le Sénégal, à la fois modernisé et islamisé, suit une évolution ambivalente selon Biaya (2002 : 341); il s'ouvre à cet hédonisme, "les plaisirs de la cité » selon l'auteur, tout en le réglementant par le véhicule de l'Islam maraboutique. Un pan de la modernité conduit ainsi à une érotisation du 
corps chez les jeunes prenant forme dans une expression culturelle provocatrice dont le fer de lance est un véritable culte du corps dévoilé entrant en rupture totale avec la vertu de la pudeur défendue par les monothéismes selon Amouzou (2009 : 51). D'autant plus que cette culture urbaine africaine de manière générale est érigée en mode de vie par la jeunesse (Biaya, 2002). Mais l'Islam le plus orthodoxe et réactionnaire vis-à-vis de l'hédonisme moderne peut imposer malgré tout le port strict des tenues vestimentaires islamiques aux femmes dans certains pays africains comme le Sénégal. "Aujourd'hui les ibaadourahmane ont installé partout dans le pays des mosquées et des écoles et éduquent les gens; ce sont d'ailleurs leurs filles que l'on voit se voiler (Seck, 2010 : 141) ». Cette discipline du voile s'opposerait de manière générale à leur émancipation si l'on en suit le discours de Watson (1997), et peut-être aussi dans une moindre mesure pour le Christianisme en Afrique, celui-ci entrant également en opposition avec ces valeurs modernes hédonistes (Kuate-Defo, 2000).

Cette hédonisation de la sexualité va de pair avec une hédonisation du corps - toutes deux très controversées - par le développement des tenues vestimentaires modernes qui mettent en avant les régions sexuées corporelles (Desjardin, 1995 : 4). La modernité fantasme des formes corporelles anti-nature (Fischler 2001 : 357 ; 361), par exemple, désirer un buste large lorsque l'on est mince ou des grosses fesses lorsque l'on est menu, pour évidemment les exhiber. Comme l'avance Ndiaye (2009) au Sénégal, «avoir la «taille de guêpe », la «forme Coca Cola», selon le champ lexical argotique wolof», c'est "disposer de hanches aplaties et d'une poitrine bien pleine ». Les jeunes garçons quant à eux veulent acquérir un corps noueux, musclé de manière sèche (Neveu-Kringelbach, 2007), jusqu'à développer en Afrique du Sud une nouvelle nosologie en santé mentale : «muscle dysmorphia » (Hitzeroth et al., 2001). Ces réalités se retrouvent dans les grandes villes d'Afrique Centrale comme Yaoundé (Essomba, 2006) ou Libreville, Tonda (2007) avançant que «le souci du corps beau et élégant s'inscrit en porte-à-faux du souci de « solidarité », les reproches se faisant alors clairement entendre : une fille ou un garçon "ne pense qu'à lui-même, ne pense qu'à s'habiller », à « faire le beau », donc à jouer son corps sur le billard urbain ».

Pourtant, ces critères esthétiques exigeants entrent en rupture avec les critères esthétiques que l'on retrouve dans de nombreuses sociétés villageoises d'Afrique. Une valorisation d'un corps fort et robuste pour pouvoir travailler dans les champs (Faye, 2002), mais aussi d'un léger embonpoint signe de vitalité et de prospérité étaient de mise (De Garine, 1962 ; Warnier, 1993 ; Cohen et al., 2013), et de toute évidence bien différents de ce qui est valorisé avec la modernité.

En effet, cette esthétique corporelle moderne exigeante se véhiculant dans l'ensemble de 
l'Afrique urbaine (Mazzocchetti, 2010), Le Breton (2005 : 157) en a initialement fait la constatation en Occident. Selon lui, depuis la fin des années soixante, et avec une extension grandissante, un nouvel imaginaire grandissant sur le corps a pris un essor et conquis des domaines de pratiques et de discours jusqu'alors inédits. Il devient le territoire de la reconquête de soi, à explorer indéfiniment pour impressionner les autres, manifester d'une expression corporelle toujours plus inaccessible biologiquement. Ceci impose une situation où la compétition entre les individus, à travers leur corps devant toujours se perfectionner, devient de plus en plus ardue.

Dans un contexte de mutation sociale prégnant des sociétés africaines par leur modernisation, les Africains construisent leurs critères esthétiques, entre valeurs traditionnelles et modernes, en interaction avec le rapport dynamique qu'ils entretiennent avec la sexualité entre hédonisme et reproduction. En prenant le cas du Sénégal, du Cameroun et du Burkina Faso, ce travail permettra de mettre en évidence un nouveau rapport au corps qui émerge en Afrique, en opposition avec les valeurs des aînés toujours persistante, impliquant une mondialisation envisageable des névroses modernes de l'image du corps.

\section{UNE MUTATION DES PRATIQUES SEXUELLES}

\section{Une nouvelle sexualité hédoniste axée sur la recherche de plaisir}

Avec la modernisation de l'Afrique, la vie de couple «par amour» non-arrangée par les parents, et souvent hors mariage s'instaure (Thiriat, 1999). L'une des modalités fondatrice de l'existence du couple - et pas la moindre - s'impose : l'hédonisme sexuel prononcé. La modernité, à travers ses médias et TIC, véhicule de nouvelles pratiques de la sexualité, en ville comme au village, non conformes aux manières de faire traditionnelles, que de nombreux condamnent à l'instar de Kobelembi (2005). Ces nouvelles approches de la sexualité créent le décalage intergénérationnel certainement le plus intense, et les médias modernes semblent avoir un rôle à jouer majeur dans le creusement de ce fossé entre jeunes et plus âgés (Amouzou, 2009 : 44). Ils parlent, surtout les plus âgés, d'une véritable perversion de la société car selon eux, les jeunes seraient devenus obnubilés par la recherche de plaisir sexuel en reproduisant des comportements et pratiques qu'ils découvrent dans les feuilletons occidentaux (télénovelas et autres émissions de ce genre) et les films pornographiques. "C'est sans doute l'influence de ces médias qui est à l'origine de la modification de la signification de l'acte sexuel chez les jeunes. La recherche du plaisir est devenue la raison 
principale des rapports sexuels. Ainsi les jeunes sont de plus en plus exposés aux images des télévisions étrangères qui n'ont pas toutes à cœur de préserver les mours de ceux qui les regardent (Kobelembi, 2005) ». L'ouverture vers des phases préliminaires et une variabilité de positions sexuelles, apparemment inconnues dans les traditions villageoises, seraient perçues par ceux qui restent «traditionnels» comme des attitudes anti-nature, anti-reproductives, immorales, addictives et pathologiques vis-à-vis du plaisir sexuel (Image 1).

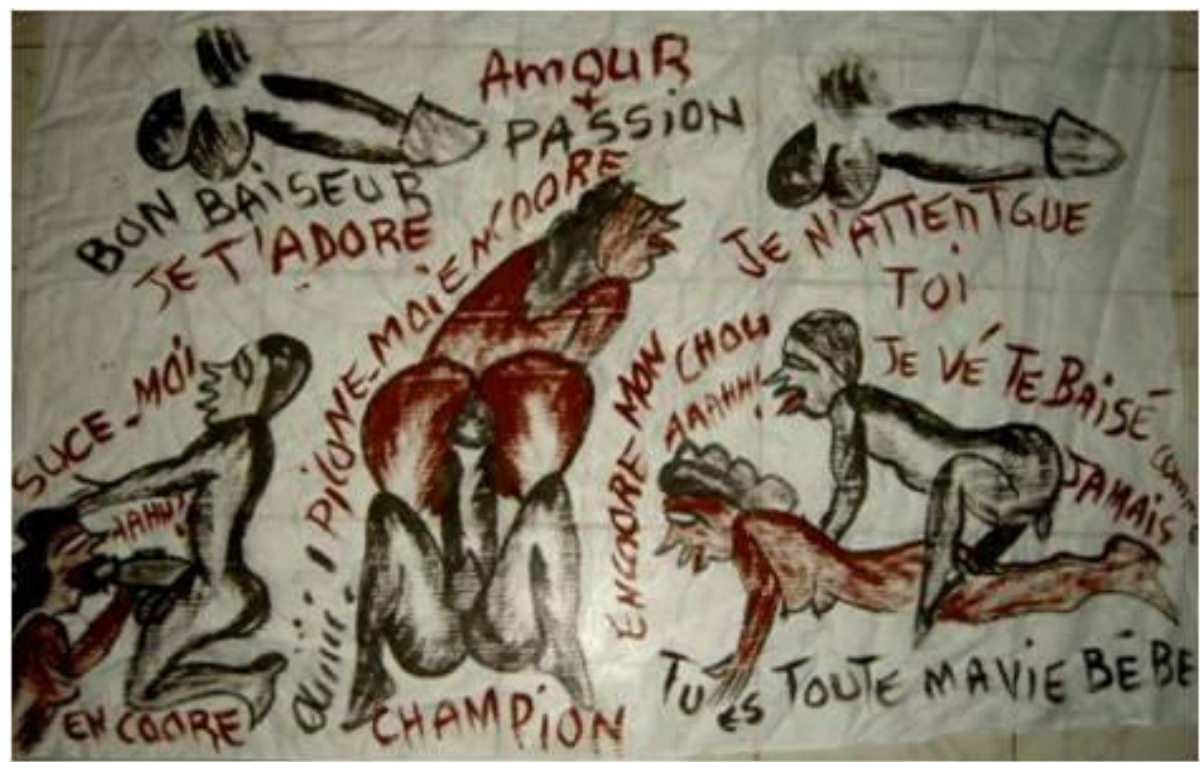

Image 1. Pagne sexuel au marché HLM à Dakar ${ }^{1}$

Marie-Thérèse, sénégalaise, fille, 24 ans, universitaire, étudiante, sérère, chrétienne :

«Avec les films pornos, il y a des positions que l'on voit. On discute entre filles de ces choses-là. C'est négatif. Eux [les occidentaux] le font car c'est leur culture mais nous, nous ne connaissons pas ça. Quelles conséquences sur la santé de la personne cela peut provoquer? Des déformations aux nouveau-nés ? C’est plutôt les « jeunes jeunes » car à 25 ans on est vraiment mature. Les minetteslà, nos toutes petites sœurs de 16,17 ans ce qu'elles font..., nous à cet âge, on ne faisait pas ça ».

Doudou, sénégalais, 23 ans, secondaire, artiste/comédien, wolof, musulman :

«Le porno, si tu vois ça, tu as envie de découvrir. La masturbation c'est une maladie. Vraiment c'est une chose absurde quoi. Exhiber ses parties intimes pour avoir du plaisir seul. C'est comme la pédophilie. Et je juge que c'est très mauvais. C'est une sorte de perversion. Il y a des hommes, ils ne peuvent plus s'en départir. C'est devenu une seconde nature, c'est eux, c'est leur vie ».

Michel, camerounais, 29 ans, universitaire, comptable, Béti, chrétien :

«Les gens voient les films pornos et sont influencés. Mais ceux qui vivent dans les villages et qui 
n’ont pas accès à l'électricité, ils ne connaissent pas tout ça. C'est juste le missionnaire »

Zarata, burkinabé, 32 ans, secondaire, ménagère, Mossi, musulmane :

«Normalement, la pornographie ne devrait même pas exister. C'est irrespectueux, notamment pour les femmes. La sodomie, ce n'est pas normal. Mais les petites caresses, les préliminaires, c'est bien pour la femme, mais les hommes dans la tradition ne connaissent pas ça ».

Ils mettent en avant les valeurs de l'Islam et aussi ce qu'ils nomment « les valeurs africaines traditionnelles »- donc des valeurs plutôt animistes -, pour condamner ces nouvelles manières de faire qu'ils jugent aberrantes. Des auteurs ont montré en effet combien dans différentes «traditions africaines », l'acte sexuel en lui-même ne suit pas un protocole aussi diversifié (Echard, 1985). Ce nouveau rapport à la sexualité semble complètement d'actualité, et en déphasage total avec la génération des parents encore sous l'influence des valeurs issues des traditions villageoises. En effet, pour Kobelembi (2005), "on assiste aujourd'hui à un recul des valeurs traditionnelles qui ont jadis gouverné les attitudes et les comportements des parents dans leur jeunesse ». Ainsi, Tadele (2003) constate auprès de jeunes garçons urbains éthiopiens, que ces «nouvelles pratiques » sont très mal vues, perçues comme anormales et indécentes.

\section{Un plaisir sexuel peu développé dans la «tradition »}

Les pratiques de la sexualité dans différentes traditions villageoises sont très différentes des pratiques sexuelles modernes. L'homme et la femme ne pratiquent exclusivement que le missionnaire, le rapport est bref, rapide, sans préliminaires. «Ca se faisait naturellement hein. Naturellement, c'est clair; la femme est couchée, l'homme vient et monte dessus, et c'est tout. Ça, c’est naturel (El Hadj, sénégalais, 46 ans, universitaire, agent de sécurité, wolof, musulman) ». Pour eux, c'est le rapport le plus évident, qui va de soi. «La position normale tout le monde le sait [Rire] (Léna, sénégalaise, 22 ans, primaire, sans activité, sérère, musulmane) ». Elle s’effectue bien souvent «à sec », les jeunes filles étant «initiées au « sexe sec» (Sow et Bop, 2004 : 98) », sans caresses ou baisers. "Quand une femme excitée mouille durant le rapport sexuel, cela soulève les soupçons de l'homme, qui pense qu'elle n'est pas propre ou même qu'elle perd des urines (Sow et Bop, 2004 : 98) ». Personne ne doit voir le sexe de l'autre, la lumière étant éteinte "parce que cela leur a été enseigné et inculqué ainsi, et qu'ils n'osent pas déroger aux règles et normes établies ». Ainsi, les femmes comme les hommes, et même plus que les hommes, en viennent naturellement à préférer avoir des relations sexuelles dans l'obscurité pour éviter d'affronter la dimension visuelle du plaisir sexuel. 
Adama (rural), sénégalaise, 46 ans, aucun niveau, ménagère, sérère, musulmane:

« Dans l'Islam il y a des choses à faire avec pudeur. La nuit et le jour, ça résume tout. Aujourd’hui il y a les embrassades, les films porno, mais moi je n'en ai jamais vu. C'est un grand péché de suivre ce genre de film. C'est de l'animalité. Tout doit se faire dans la discrétion et dans la pudeur. Si un homme s'habitue à observer le sexe de sa femme, avant sa mort il sera aveugle ».

"Certaines positions et pratiques jugées convenables sont adoptées tandis que d'autres sont rejetées parce qu'on les trouve « bestiales » ou «non naturelles » (Sow et Bop, 2004 : 103)». L'objectif est l'émission efficiente de sperme (Echard, 1985) pour favoriser la dimension reproductive, fondamentale dans les sexualités traditionnelles africaines selon Ombolo (1991 : 369). "C'est pourquoi l'érotisme sous toutes ses formes ainsi que toutes les façons de s'exploiter ou d'exploiter l'autre sur le plan sexuel sont restés ignorés de l'Afrique Noire traditionnelle et ne pouvaient du reste que difficilement investir sa mentalité tout le temps qu'elle aurait conservé sa philosophie originelle de l'être et le système éthique qu'elle implique ». De plus, le rapport sexuel ne se déroule jamais la journée car la vie conjugale commence au crépuscule, uniquement dans la case des parents, où les enfants en bas âge sont bien souvent à proximité. Ainsi, la société offrant un espace social très limité à la sexualité, la promiscuité entre mariés et le plaisir qu'ils peuvent s'offrir sont donc relativement limités aussi.

Francis, camerounais, 27 ans, secondaire, technicien cybercafé, Bassa, chrétien :

«La sodomie, c'est ignoble, mais la fellation, je peux dire que ça fait partie du plaisir. Aujourd'hui, on est plus éveillé sur certaines choses, on prend le temps d'explorer, comme les préliminaires. Même les parents d'aujourd'hui découvrent certaines choses, mais ceux de la génération d'avant, pas du tout. L'homme montait sur la femme, faisait ce qu'il avait à faire et c'est tout ».

Ibrabim, burkinabé, 32 ans, secondaire, électricien, Mossi, musulman :

«A la génération de mon père, personne ne connaissait cette sexualité que l’on voit dans la pornographie. Il y a même des gens aujourd'hui au Burkina qui ne connaissent pas ça. Chez nous, les vieux expliquaient aux jeunes mariés comment il faut faire. C'est très simplement aller se coucher, il n'y avait pas tout ça. Chaque jour on dit que c'est l'homme qui doit être sur la femme ». 
Elisa, burkinabé, 26 ans, universitaire étudiante, Mossi, chrétienne :

«Avant le corps n'était pas objet sexuel. On ne connaissait pas la pornographie, comme par exemple les Mossis. Le sexe c'était un peu nul, aujourd'hui il y a des nouveaux trucs, des jeux sexuels, des préliminaires. Avant, c'était le missionnaire et c'était rapide ».

Tout ce qui vient de l'Occident est majoritairement condamné pour ce qui concerne la sexualité. On considère que ces nouvelles pratiques sont avilissantes car anti-nature et antireproductives puisque morbides pour la progéniture et conduisant à l'impuissance. "En effet, dans l'imaginaire sahélo-soudanien, toute quête de satisfaction sexuelle par la pénétration du pénis dans un autre organe que le vagin reste culturellement considérée comme un acte contre-nature (Biaya, 2001)». L'idée de rechercher du plaisir personnel dans la sexualité est perçue comme pathologique. C'est à croire qu'ils font le sexe parce qu'il faut le faire (Hanry, 1970 : 150), comme l'a avancé l'un de nos jeunes sénégalais, «à cette époque-là, la sexualité était quasi inexistante (Adama, sénégalais, 22 ans, universitaire, étudiant, soninké, musulman) ». Elle est un capital pour le groupe, mis au service de celui-ci. «Il est indiscutable que la clitoridectomie tout en favorisant le passage du plaisir narcissique (masturbation solitaire) au plaisir hétérosexuel donc « social » du coüt confirme-la «fonction maternelle » du vagin, instrument au service exclusif de l'espèce. De même, l'ablation du prépuce souligne la séparation définitive du fils par rapport à sa mère, donc exprime la maturité sexuelle de l’homme faisant de lui désormais un procréateur (Thomas, 1990) ». Comme l'on affirmé les enquêtés, il y a une volonté sociale presque consciente de s'éloigner au plus possible de toute recherche intense de satisfaction sexuelle. La sexualité semble plus être un devoir qu'une envie pour eux. "L'homme doit niquer l'épouse chaque fois qu'il lui est possible de le faire, c'est-à-dire la nuit, dans une habitation, en dehors des périodes de menstruation (Echard, $1985)$ », pour des fins reproductives. C'est pour cela que bien souvent, ils considèrent les occidentaux pervers : "Ils ne pensent qu'au sexe, ils aiment trop le sexe, avec eux, il n'y a que le sexe », même si certains se laissent aller à découvrir.

Tapha, sénégalais, 25 ans, universitaire, étudiant, wolof, musulman :

«Ah, quand tu prends la femme de derrière, c'est comme la brouette quoi [rire !!Il fait un signe avec ses mains pour montrer qu'il tient quelque chose devant lui en rigolant]. C'est du n'importe quoi ce truc, y a que les Blancs pour faire ça franchement. Mais évidemment, ici les gens commencent à le faire, j'entends que dans telle chambre, ils ont baisé et qu'ils ont fait ça. Mais c'est trop nouveau, ils 
essayent, juste pour voir, mais moi je n'en ai pas besoin ».

Karim, burkinabé, 18 ans, aucun niveau, musicien, bobo, musulmam/ animiste :

«Moi je suis à la mode, je couche avec les femmes comme les Blancs. La position de derrière et tout [rire !!], c'est la classe ».

Dorothée, camerounaise, 46 ans, secondaire, cuisinière restaurant, Béti, chrétienne:

«Le mariage, c'est une fille, un garçon; on te dit: «voilà ta femme, voilà ton mari » et ils grandissent comme ça.... Tu fais l'enfant c'est tout... c'est l'homme qui monte et c'est tout ».

"La fonction sexuelle chez les Pahouins apparaît comme un mal foncier ; mais si c'est un mal nécessaire par lequel il faut passer pour les besoins de la procréation, la valeur suprême sociale, il convient naturellement d'en limiter les dégâts en l'ordonnant exclusivement à cette seule valeur positive qu'il apporte. Dès lors il devient clair qu'un coüt qui n'aurait pas pour raison la recherche de cette valeur, soit en elle-même, soit dans la légitimité, soit objet interdit ; l'Afrique Noire est restée dans son ensemble étrangère aux modèles socioculturels de liberté et de perversions sexuelles (Ombolo, 1991 : 369) ». S'il n'y a pas de variabilité de positions (sexe), ni même de baisers, câlins, enlacements ou caresses (amour), c'est que les notions de plaisir et d'amour, fondatrices de l'instance du couple, n'existent pas dans les traditions villageoises, en tout cas pas telles que nous l'entendons, entre hommes et femmes. «Les bisous, les baisers, les embrassades, les grands-parents ne connaissaient pas. Mais aujourd'hui l'homme et la femme se font des bisous, ils s'embrassent, la femme se met au-dessus. Les gens font les rapports sexuels en étant même debout; tu vois un homme baiser sa petite amie contre un mur (Modou (rural), sénégalais, 22 ans, aucun niveau, tôlier, wolof, musulman)». L'histoire de l'Occident a donc orienté l'histoire des pays africains, précisément pour ce qui concerne la sexualité. «Les positions par ci par là, ça c’est la modernité qui a amené ça, ce sont les films pornos et autres. Si ce n'était pas la modernité, moi je pense que les gens allaient rester comme ça, ils n’allaient pas découvrir certaines positions (El Hadj)».

«Le mariage, qu'il soit coutumier, religieux ou civil, comporte notamment la reconnaissance de l'obligation d'entretenir une vie sexuelle active, selon certaines règles. [...] Cette obligation de vie sexuelle active a un double objectif. L'un - la maternité - est reconnu; l'autre - le plaisir - est en général passé sous silence ou perçu comme un à-côté. Il est considéré comme un devoir pour les femmes d'avoir des enfants dans le cadre du mariage, au point que la stérilité constitue un véritable drame. Par contre, la dimension propre au plaisir 
de la vie sexuelle est estimée au mieux comme un agrément supplémentaire, mais non indispensable à la vie conjugale (Sow et Bop, 2004 : 105) ». Ainsi, nombre d'enquêtés n'arrivent même pas à concevoir le fait que les Occidentaux aient pu inventer de telles positions et manières de faire. «Le fait qu'une femme suce le sexe de l'homme, c'est horrible. Moi je n’ai jamais pensé qu'une telle chose pouvait être avant que je vois ça (Macoumba). Le sexe n’est pas fait pour être sucé quoi (Demba (rural), sénégalais, 20 ans, secondaire, élève, sérère, musulman) ».

Ils sont tellement bouleversés par ces mutations qu'ils diabolisent ces pratiquent, en y prêtant des conséquences désastreuses. "Je crois que ce n’est pas bon ni pour l’homme ni pour la femme ni pour les enfants qui vont naître (Macoumba (rural), sénégalais, 24 ans, primaire, commerçant, wolof, musulman) ». Le plus ironique, c'est que certains en arrivent même à penser qu'en fin de compte, ces pratiques sexuelles -banales en Occident - seraient du bluff, pour le spectacle, pour faire vendre les films et qu'elles se ne pratiquent pas réellement dans le quotidien des occidentaux. "Je vois que c'est très dangereux pour nos jeunes parce qu'ils ne sont pas conscients du fait que ceux qui font ces films sont payés pour l'exécuter de la sorte, mais eux ne la pratiquent pas de cette manière (Ndèye Diayté (rural), sénégalaise, 51 ans, primaire, commerçante, peul, musulmane) ». C'est le type de plaisir en lui-même - libidinal et affectif qui a une nature différente. "S'embrasser sur la bouche, on ne connaissait pas. Dans notre culture Mossi, on dit qu'ils se mangent la bouche. La bouche, ça sert pour manger la nourriture (Ibrahim) ». « Pour eux, les baisers, les caresses c'était une perte de temps, un truc pour les Blancs (Elisa)»

Nous parlons de «traditions » pour ce qui concerne la sexualité - ou même de sexualité traditionnelle - car le discours est très homogène sur cette question, malgré les différences ethniques et religieuses, et se retrouve dans d'autres pays d'Afrique (Tamale, 2008 ; Coulibaly et al., 2015). Les plus vieux enquêtés rendent bien compte de cette volonté d'économiser l'énergie, en l'occurrence le sperme. Il ne faut pas la/le dilapider, appréhension qu'ils expriment par leur joie de ne s'être ouverts à la vie sexuelle qu'à trente ou quarante ans, que l'on retrouve au Cameroun. " "Mener une vie très rangée», "ne pas gâcher sa jeunesse », "prendre soin de soi-même », n'est-ce pas s'épargner, accumuler, pour remplir la tirelire vitale à des fins ultérieures d'engendrement. [...] Comment mieux suggérer que plus le mariage est tardif, plus la substance a eu le temps de se concentrer? (Warnier, 1993 : 131)». «Si le jeune joue avec sa jeunesse, au mariage, il ne pourra même pas avoir d'enfants solides. Les vieux avant de connaître la femme allaient jusqu’à la trentaine, au mariage 
(Macoumba)». «Les jeunes vieillissent vite aujourd'hui, car la vie va trop vite, les jeunes découvrent trop tôt. Quand tu fais le sexe, après tu es faible, ça te fait vieillir vite (Ibrahim)».

Cette posture animiste s'exprime chez les vieux par leur condamnation stricte de la masturbation, c'est-à-dire une dilapidation inutile. "L'engendrement est perçu comme une dépense d'énergie, un travail. La parcimonie n'affecte pas seulement la dépense monétaire et la consommation. L'austérité des mours sexuelles bamiléké soulignée par Ongoum (1985: 286) est enracinée dans le sentiment de la rareté, de l'économie des forces, de leur gestion au plus près des ressources disponibles (Warnier, 1993 : 130) ». Une méfiance à l'égard du désir sexuel extrême et des pratiques abusives qu'il pourrait impliquer s'exprime. "L'Africain traditionnel le faisait par devoir et par nécessité (Moussa, sénégalais, 24 ans, secondaire, sans activité, wolof, musulman) ». «Ils le faisaient parce que c'était une obligation de coucher avec sa femme (Amadou (rural), sénégalais, 24 ans, universitaire, étudiant, peul, musulman) ». Les vieux pensent en effet que les femmes peuvent leur voler leur énergie vitale par le coït, donc il faut limiter son usage à sa rentabilité : la procréation. «Les ancêtres savaient que la femme (le sexe) est un poison pour l'homme. Ça diminue vraiment la puissance de l'homme donc il ne faut pas en abuser (Mamadou (rural), sénégalais, 54 ans, aucun niveau, cultivateur/guérisseur, sérère, musulman)».

\section{UNE EXIGENCE ACCRUE EMERGE SUR LE CORPS}

Cette importance accordée à l'apparence physique, concomitante à une importance accordée au plaisir sexuel - à l'instar de l'Europe depuis les années 60 (Meidani, $2007: 36$ ) - conduit à une exigence esthétique du corps qui pourrait potentiellement ouvrir les populations, notamment les plus jeunes occidentalisés, aux névroses corporelles modernes de l'image corporelle (Cash et al., 2004) : troubles alimentaires, dysmorphies corporelles, etc... Les critères de beauté deviennent de plus en plus inaccessibles, notamment vis-à-vis de la corpulence et de la forme corporelles, les stratégies pour rendre son corps parfait - sans les effets de la nature - esthétiquement se développent. De par ce modèle esthétique axé sur une exigence accrue de l'apparence, de nouveaux processus de stigmatisation émergent. Les individus non désirables sexuellement - et «moches »-, c'est-à-dire ne correspondant pas à cette beauté de l'extérieur proposée par la modernité, se voient soumis à différents écueils dans leur volonté d'accéder à la vie sexuelle et affective (Testard-Vaillant, 2008). Il faut être taille coca-cola pour les filles, "hanches aplaties et [...] poitrine bien pleine (Ndiaye, 2009)»; ceene [musclé] pour les hommes, «jeune, musclé (Baller, 2007)», correspondre à ces modèles modernes exigeants de la forme corporelle, sinon on ne se sent plus accepté par 
les autres, fait que constate aussi Neveu Kringelbach (2007) à Dakar.

\section{El Hadj:}

«Le sein n'était pas vu comme une partie sexuelle. Les filles aujourd'hui y accordent une très grande importance. On voit des femmes qui ont un bébé, par exemple, mais elles ne veulent même pas l'allaiter. [Rires .]. Elles préfèrent chercher un biberon pour mieux maintenir leurs seins ».

André, sénégalais, 26 ans, universitaire, étudiant, diola, chrétien :

«J'ai vu des gens qui accordent tellement d'importance à leur apparence physique. Par exemple j'ai un ami qui accorde tellement d'importance à la musculation, il passe presque la nuit à faire de la musculation. Ce n'est pas mon cas, je n'en fais pas une obsession. J'en fais mais le juste milieu. Je discute des fois avec lui sur ça, mais il me dit souvent que s'il avait ma corpulence (mince), il n’allait plus sortir de sa chambre. Il se sentirait gêné, complexé. Alors que je sais que je suis normal».

\section{Ibrabim :}

«Les gens avant n’avaient pas ce temps de regarder la femme comme ça. Aujourd'hui, tu regardes tout, jusque même là où tu vas aller. Les habillements sexuels y sont pour beaucoup. Aujourd'hui on sélectionne la beauté, mais dans nos traditions, la beauté ne compte pas. Ce qui comptait était le comportement, le respect de ta famille. On ne regardait pas si tu étais beau ou vilain. Avant, les gens ne s'amusaient pas avec les seins, dans la manière actuelle, on dit les lolos, tout ça. Ils n'avaient pas ce temps-là. Mais nous on ne voit que ça, comme un autre produit à découvrir ».

\section{Francis :}

«Avant quand les hommes épousaient les femmes, ils cherchaient à avoir un héritier. Ils voyaient si elle pouvait se reproduire. L'apparence ce n'était pas ce que l'on voit aujourd'hui, c'était le nécessaire. Il n'y avait pas tout ce maquillage, les coiffures d'aujourd'hui. Il y avait les chignons, les tresses et c'est fini. Aujourd'hui, même si l'argent compte, les filles veulent être avec des hommes mignons. Beau, bien musclé, avec les pectoraux, qu'il soit physique, bien assis. Elles vont en discuter entre elles, comparer pour savoir laquelle a le petit ami le plus mignon, c'est une concurrence ».

Ce culte moderne du corps parfait, exempté des contraintes naturelles (Le Breton, 2005 : 229) », induit un rejet progressif de l'embonpoint, comme c'est le cas dans la modernité 
occidentale (Testard-Vaillant, 2008). Ce phénomène est observé par Sow (2002) et Ndiaye (2009) au Sénégal. Le premier auteur rend compte d'une médecine sénégalaise investie par les critères esthétiques modernes. On retrouve alors une volonté de rejeter l'embonpoint (Figure 1), chez les jeunes sénégalais les plus ouverts à la modernité, qui dépasse largement les critères de santé. En effet, à l'inverse des femmes d'âge mûr, "de plus en plus de jeunes filles sénégalaises se privent de nourriture pour devenir des " top modèles » au risque d'être pré-cachectiques (Sow2002) ». Les deux auteurs avancent que le culte de la minceur extrême tend à se généraliser sous l'influence des médias de diffusion et des TIC au point que certaines Sénégalaises frôlent l'anorexie.

Yacine (rural), sénégalaise, 55 ans, primaire, ménagère, wolof, musulmane :

«Maintenant, tu vois que les filles font des régimes alors qu'elles ne sont même pas am yaram [avoir du poids]. Elles font tout pour plaire, nous on ne connaissait pas ça »

Ndèye Diama (rural), sénégalaise, 18 ans, secondaire, élève, sérère, musulmane :

«Je ne veux pas de ces types d'homme am yaram [avoir du poids] avec un gros ventre. Je veux un homme yem [moyen], ceene [musclé] qui répond bien à mes attentes. Un homme qui peut satisfaire sexuellement ».

Francis :

«Pour les garçons aussi ça compte. Par exemple, ma copine était mince, maintenant elle a pris du poids. Le ventre et tout ça me dérange, je lui dis qu'il faut maigrir ».

Elisa :

«Avant c'était juste le pagne, il n'y avait pas tout ce modèle vestimentaire d'aujourd'hui. C'est vrai qu'une femme avec du poids était mieux perçue avant, mais si tu étais mince avec de bonnes valeurs morales, tu avais ta place dans la société. Aujourd'hui, on accorde beaucoup d'importance à l'importance. Un jeune qui est trop gros ou mal habillé, il peut être rejeté par les autres ».

Tradition [coosan]

Modernité [dund tubab]

La corpulence chez la femme

Diongoma

Driyanké

Taille-fine

La corpulence chez l'homme

\section{Birru patron}

Jaaraf

La forme chez la femme 
Figure 1. Evolution des corpulences valorisées au Sénégal

Cette évolution socioculturelle tend vers l'affirmation de l'individu narcissique au détriment de son groupe, avec l'ensemble des névroses modernes du soi (Ehrenberg, 2008 : 236) qu'elle implique - notamment les névroses de l'image de son corps (Cash et al., 2004) dans une société où l'apparence corporelle est le principal support d'expression de soi (Balandier, 2004). Les prémices de cette tendance furent déjà observées par Le Guérinel (1980) avec l'urbanisation des pays africains et les débuts de l'ère postcoloniale, et il semble évident que l'Afrique doit à partir d'aujourd'hui composer avec le développement de ces affections mentales dans les régions les plus modernisées du continent (Gitau et al., 2014).

\section{NOTES DE FIN}

${ }^{1}$ Photo d'Aurélie Fontaine.

\section{BIBLIOGRAPHIE}

AMOUZOU (E.) 2009, L'impact de la culture occidentale sur les cultures africaines, l'Harmattan, Paris.

BALANDIER (G.) 2004, Ce que « disent» le corps et le sport, Métissages 6-7. (Article publié sur http://corpsetculture.revues.org/document885.html).

BIAYA (T.K.), 2001, Les plaisirs de la ville : Masculinité, sexualité et féminité à Dakar (1997-2000), African studies review 44(2) : 71-85.

BIAYA (T.K.), 2002, Culture du loisir et culture politique, in M.C. Diop (éd.), Le Sénégal contemporain, Karthala, Paris, p. 341-352. 
CASH (T.F.), MORROW (J.A.), HRABOSKY (J.I.), PERRY (A.A.) 2004, How has body image changed? A cross-sectional investigation of college women and men from 1983 to 2001, Journal of Consulting and Clinical Psychology 72 (6): 1081-1089.

COQUERY (V.), 1988, Les populations africaines du passé, in D. Tabutin (éd.), Population et sociétés en Afrique au Sud du Sahara, l'Harmattan, Paris, p.51-72.

COULIBALY (A.) , 2015, Fécondité et nouvelles expressions de la sexualité à Bamako et en Afrique de l'Ouest, in G. Boëtsch, E. Macia, L.Gueye, Y. Jaffré (éd.), Santé et sociétés en Afrique de l'Ouest, Cnrs Editions, Paris, p.47-72.

DESJARDINS (G.), 1995, L'amour en patience: la sexualité adolescente au Québec, 1940-1960, PUQ, Québec.

DESJEUX (C.), BONNET (D.), DESJEUX (B.) 1983, Africaines, l'Harmattan, Paris.

ECHARD (N.), 1985, « Même la viande est vendu avec le sang, de la sexualité des femmes, un exemple », in N.C. Mathieu (éd.), L’Arraisonnement des femmes, EHESS, Paris, Cahiers de l'Homme, 24 : 37-60.

EHRENBERG (A.) 2008, La fatigue d'être soi : Dépression et société, Odile Jacob, Paris.

ESSOMBA (A.) 2006, Civilité publique et identités sexuelles dans les rues de Yaoundé, Polis/ R.C.S.P./C.P.S.R. 13(1-2) : 103-117.

FAYE (A.), 2002, La beauté Seereer : du modèle mythique au motif poétique, Ethiopiques 68. (Article publié sur http://www.refer.sn/ethiopiques).

GITAU TM, MICKLESFIELD LK, PETTIFOR JM, NORRIS SA (2014) Changes in Eating Attitudes, Body Esteem and Weight Control Behaviours during Adolescence in a South African Cohort. PLoS ONE 9(10): e109709.

HANRY (P.), 1970, Erotisme africain, Payot, Paris.

HITZEROTH (V.), WESSELS (C.), ZUNGU-DIRWAYI (N.), OOSTHUIZEN (P.), STEIN (D.J.) 2001, Muscle dysmorphia: a South African sample, Psychiatry and Clinical Neurosciences 55: 521523.

KOBELEMBI (F.), 2005, Le comportement sexuel des adolescents à Bangui(RCA), African Population Studies, 20(2) : 65-99.

KUATE-DEFO (B), 2000, L'évolution de la nuptialité des adolescentes au Cameroun et ses déterminants, Population 55(6) : 941-973.

LE BRETON (D.) 2005, Anthropologie du corps et modernité, PUF, Paris.

LE GUERINEL (N.) 1980, Note sur la place du corps dans les cultures africaines, Journal des africanistes 50(1) : 113-119. 
MAZZOCCHETTI (J.), 2010, À la recherche de l'homme capable... Concurrence entre femmes (Ouagadougou, Burkina Faso), Civilisations 59(1), (Article publié sur http://civilisations.revues.org/2241).

MEIDANI (A.) 2007, Les Fabriques Du Corps, Presses Univ. du Mirail, Villeneuve d'Ascq.

NDIAYE (L.) 2009, Mondialisation et cultures, Ethiopiques 82. (Article publié sur http://www.refer.sn/ethiopiques).

NGONDO A PITSHANDENGE (I.S.) 1994, Les mutations culturelles en matière de mariage et de sexualité en Afrique subsaharienne: est-ce le début d'une transition démographique ? in AIDELF (éd.), Les modes de régulation de la reproduction humaine: incidence sur la fécondité et la santé, PUF, Paris, p. 55-64.

NEVEU KRINGELBACH (H.) 2007, «Le poids du succès » : construction du corps, danse et carrière à Dakar, Politique africaine 107: 81-101.

OMBOLO (J.P.), 1991, Sexe et société en Afrique noire: l'anthropologie sexuelle béti, l'Harmattan, Paris.

SECK (A.) 2010, La question musulmane au Sénégal: essai d'anthropologie d'une nouvelle modernité, Karthala, Paris.

SOW (F.), BOP (C.), 2004, Notre corps, notre santé: la santé et la sexualité des femmes en Afrique subsaharienne, l'Harmattan, Paris.

TADELE (G.), 2003, Survivre dans la rue: sexualité et VIH/sida parmi les jeunes gens de la rue de Diessi, Ethiopie, Bulletin du Codesria, 2-3-4 : 109-118.

TAMALE (S.), 2008, LE POUVOIR DU PLAISIR : Erotisme, sensualité et « secrets de femmes » chez les Baganda, in A. Cornwall, S. Jolly (éd.), Au Cœur de l'importance de la sexualité, ARSRC, Lagos, IDS Bulletin 37(5) : 146-158.

TESTARD-VAILLANT (P.), 2008, Le diktat des apparences, Le Journal du CNRS, 222-223 : 19-21.

THIRIAT (M.P.) 1999, Les unions libres en Afrique subsaharienne, Cahiers québécois de démographie 28(1-2) : 81-115.

THOMAS (L.V.) 1990, Corps et société: le cas négro-africain, Cahiers des religions africaines 24(47): 193-214.

TONDA (J.) 2007, Entre communautarisme et individualisme : la "tuée tuée", une figure-miroir de la déparentélisation au Gabon, Sociologie et sociétés 39(2) : 79-99.

WARNIER (J.P.) 1993, L'esprit d'entreprise au Cameroun, Karthala, Paris.

WATSON (J.), 1997, Unruly Bodies: Autoethnography and Authorization in Nafissatou Diallo's "De Tilène au Plateau (A Dakar Childhood)", Research in African Literatures, 28(2): 34-56. 